Departement für Nutztiere, Abteilung für Schweinemedizin der Vetsuisse-Fakultät Universität Zürich

Direktor: Prof. Dr. Dr. h.c. med. vet. Ueli Braun

Arbeit unter wissenschaftlicher Betreuung von

PD Dr. med. vet. Xaver Sidler, FVH

\title{
Modifizierte Dysenterie-Teilsanierung in einem Kernzuchtschweinebetrieb
}

\author{
Inaugural Dissertation \\ zur Erlangung der Doktorwürde der \\ Vetsuisse-Fakultät Universität Zürich
}

vorgelegt von

\section{Rolf Figi}

\author{
Tierarzt \\ von Glarus Süd, Glarus
}

genehmigt auf Antrag von

PD Dr. med. vet. Xaver Sidler, FVH, Referent 
Departement für Nutztiere, Abteilung für Schweinemedizin der Vetsuisse-Fakultät Universität Zürich

Direktor: Prof. Dr. Dr. h.c. med. vet. Ueli Braun

Arbeit unter wissenschaftlicher Betreuung von

PD Dr. med. vet. Xaver Sidler, FVH

\title{
Modifizierte Dysenterie-Teilsanierung in einem Kernzuchtschweinebetrieb
}

\author{
Inaugural Dissertation \\ zur Erlangung der Doktorwürde der \\ Vetsuisse-Fakultät Universität Zürich
}

vorgelegt von

\section{Rolf Figi}

\author{
Tierarzt \\ von Glarus Süd, Glarus
}

genehmigt auf Antrag von

PD Dr. med. vet. Xaver Sidler, FVH, Referent 


\section{Inhaltsverzeichnis}

Abstract

Zusammenfassung 2

Publikationsartikel 3

Einleitung 3

Tiere, Material und Methoden $\quad 4$

Vorgeschichte 4

Betrieb 4

Sanierungskonzept 4

Kotuntersuchung und Überwachung des Sanierungserfolges 6

$\begin{array}{ll}\text { Kosten der Sanierung } & 6\end{array}$

Nutzen der Sanierung 6

$\begin{array}{ll}\text { Ergebnisse } & 6\end{array}$

Labor 6

$\begin{array}{ll}\text { Kosten der Sanierung } & 7\end{array}$

Nutzen der Sanierung $\quad 7$

Diskussion 8

Schlussfolgerungen 9

Dank 9

$\begin{array}{ll}\text { Literatur } & 10\end{array}$

$\begin{array}{ll}\text { Korrespondenz } & 10\end{array}$

Lebenslauf 
Vetsuisse-Fakultät Universität Zürich (2014)

Rolf Figi

Departement für Nutztiere, Abteilung für Schweinemedizin, xsidler@vetclinics.uzh.ch

\section{Eradication of swine dysentery as modified partial depopulation in a nucleus sow breeding farm}

Abstract

On a Swiss nucleus sow breeding farm with 170 sows and 600 gilts / fatteners, an eradication of swine dysentery as modified partial depopulation was conducted in stages over a period of 12 weeks in 2011 after Brachyspira (B.) hyodysenteriae was detected in the herd. In addition to administering oral medication $(8.1 \mathrm{mg}$ tiamulin per $\mathrm{kg}$ body weight) for 4 weeks to the pigs remaining on the farm, all stables were cleaned thoroughly and the residual slurry was disinfected with Alzogur® $(3 \mathrm{l} / \mathrm{m} 3)$ while the surfaces were disinfected with Venno Vet 1 Super $\AA$ (1.5\%). At the same time rodent and fly control was intensified. Upon completion of the eradication programme, the farm was monitored for 6 months by carrying out fecal swab analyses of pigs with diarrhea. All fecal samples were negative for $B$. hyodysenteriae. The costs of the eradication amounted to approximately CHF 104,500 . The eradication yielded significantly higher live daily weight gain $(+23.8 \mathrm{~g}, \pm 10.1 \mathrm{~g}$, $\mathrm{P}<0.0001$ ). This improved performance resulted in an additional economic benefit of CHF 18,500 per year.

Keywords: eradication, Brachyspira hyodysenteriae, swine dysentery, economic feasibility, sow breeding farm 
Vetsuisse-Fakultät Universität Zürich (2014)

Rolf Figi

Departement für Nutztiere, Abteilung für Schweinemedizin, xsidler@vetclinics.uzh.ch

\section{Modifizierte Dysenterie-Teilsanierung in einem Kernzuchtschweinebetrieb}

Zusammenfassung

In einem Brachyspira (B.) hyodysenteriae positiven Schweizer Kernzuchtbetrieb mit 170 Muttersauen und 600 Jungsauenaufzucht- / Mastplätzen wurde 2011 eine rollende Dysenteriesanierung über die Dauer von 12 Wochen durchgeführt. Neben einer vierwöchigen oralen Medikation (8.1 mg Tiamulin pro kg Körpergewicht) der auf dem Betrieb verbleibenden Schweine wurden sämtliche Stallungen gründlich gereinigt sowie die Restgülle mit Alzogur ${ }^{\circledR}\left(3 \mathrm{l} / \mathrm{m}^{3}\right)$ und die Stalloberflächen mit Venno Vet 1 Super $^{\circledR}(1.5 \%)$ desinfiziert. Gleichzeitig wurde die Schadnager- und Fliegenbekämpfung intensiviert. Nach der Sanierung wurde der Betrieb während 6 Monaten mittels Untersuchung von Kottupfern von Durchfalltieren überwacht. In sämtlichen Kotproben konnten nach der Sanierung keine B. hyodysenteriae nachgewiesen werden. Die Sanierungskosten beliefen sich auf etwa CHF 104'500.-. Die Sanierung führte zu signifikant höheren Lebendtageszunahmen $(+23.8 \mathrm{~g}$, $\pm 10.1 \mathrm{~g}, \mathrm{P}<0.0001)$. Diese Leistungsverbesserung erbrachte einen jährlichen Mehrgewinn von CHF 18‘500.-.

Schlüsselwörter: Sanierung, Brachyspira hyodysenteriae, Schweinedysenterie, Wirtschaftlichkeit, Schweinezuchtbetrieb 


\title{
Modifizierte Dysenterie-Teilsanierung in einem Kernzuchtschweinebetrieb
}

\author{
R. Figi ${ }^{1}$, F. Goldinger ${ }^{2}$, E. Fuschini ${ }^{3}$, S. Hartnack ${ }^{4}$, X. Sidler ${ }^{1}$
}

${ }^{1}$ Departement für Nutztiere, Abteilung Schweinemedizin, Vetsuisse-Fakultät, Universität Zürich, ${ }^{2}$ Tezet AG, Mühlheim, ${ }^{3}$ SUISAG SGD, Sempach, ${ }^{4}$ Abteilung für Veterinärepidemiologie, Vetsuisse-Fakultät, Universität Zürich

\begin{abstract}
Zusammenfassung
In einem Brachyspira (B.) hyodysenteriae positiven Schweizer Kernzuchtbetrieb mit 170 Muttersauen und 600 Jungsauenaufzucht-/Mastplätzen wurde 2011 eine rollende Dysenteriesanierung über die Dauer von 12 Wochen durchgeführt. Neben einer vierwöchigen oralen Medikation (8.1 mg Tiamulin pro kg Körpergewicht) der auf dem Betrieb verbleibenden Schweine wurden sämtliche Stallungen gründlich gereinigt sowie die Restgülle mit Alzogur ${ }^{\circledR}\left(3 \mathrm{l} / \mathrm{m}^{3}\right)$ und die Stalloberflächen mit Venno Vet 1 Super ${ }^{\circledR}(1.5 \%)$ behandelt. Gleichzeitig wurde die Schadnager- und Fliegenbekämpfung intensiviert. Nach der Sanierung wurde der Betrieb während 6 Monaten mittels Untersuchung von Kottupfern von Durchfalltieren überwacht. In sämtlichen Kotproben konnten nach der Sanierung keine $B$. hyodysenteriae nachgewiesen werden. Die Sanierungskosten beliefen sich auf etwa CHF 104'500.-. Die Sanierung führte zu signifikant höheren Lebendtageszunahmen $(+23.8 \mathrm{~g}, \pm 10.1 \mathrm{~g}$, $\mathrm{P}<0.0001)$. Diese Leistungsverbesserung erbrachte einen jährlichen Mehrgewinn von CHF 18'500.-.
\end{abstract}

Schlüsselwörter: Sanierung, Brachyspira hyodysenteriae, Schweinedysenterie, Wirtschaftlichkeit, Schweinezuchtbetrieb

\section{Eradication of swine dysentery as modified partial depopulation in a nucleus sow breed- ing farm}

On a Swiss nucleus sow breeding farm with 170 sows and 600 gilts/fatteners, an eradication of swine dysentery as modified partial depopulation was conducted in stages over a period of 12 weeks in 2011 after Brachyspira (B.) hyodysenteriae was detected in the herd. In addition to administering oral medication ( $8.1 \mathrm{mg}$ tiamulin per $\mathrm{kg}$ body weight) for 4 weeks to the pigs remaining on the farm, all stables were cleaned thoroughly and the residual slurry was disinfected with Alzogur ${ }^{\circledR}\left(3 \mathrm{l} / \mathrm{m}^{3}\right)$ while the surfaces were disinfected with Venno Vet 1 Super ${ }^{\circledR}(1.5 \%)$. At the same time rodent and fly control was intensified. Upon completion of the eradication programme, the farm was monitored for 6 months by carrying out fecal swab analyses of pigs with diarrhea. All fecal samples were negative for B. hyodysenteriae. The costs of the eradication amounted to approximately $\mathrm{CHF}$ 104 '500. The eradication yielded significantly higher live daily weight gain $(+23.8 \mathrm{~g}, \pm 10.1 \mathrm{~g}, \mathrm{P}<0.0001)$. This improved performance resulted in an additional economic benefit of CHF 18,500 per year.

Keywords: eradication, Brachyspira hyodysenteriae, swine dysentery, economic feasibility, sow breeding farm

\section{Einleitung}

Brachyspira (B.) hyodysenteriae ist ein gram-negatives, obligat anaerobes Schraubenbakterium (Spirochät) und Verursacher der Schweinedysenterie. Klinisch manifestiert sich die Erkrankung nach einer Inkubationszeit von üblicherweise 10-14 Tagen in einem akuten wässrigbreiigen bis schleimig-blutigen Durchfall (Hampson, 2012). Die Erkrankung kann unter günstigen Haltungsund Umweltbedingungen auch latent verlaufen, wobei der Erreger in der Dickdarmschleimhaut persistieren kann (Waldmann, 1992). Treten belastende Stressfaktoren auf, können akute klinische Symptome ausgelöst

werden (Hampson, 2012). Wärme reduziert die Tenazität von $B$. hyodysenteriae erheblich, während im flüssigen Milieu die Infektiosität länger andauert (Heinritzi, 2002). Unter experimentellen Bedingungen ist $B$. hyodysenteriae in Schweinekot bis 112 Tage überlebensfähig (Boye et al., 2001). Als Infektionsquellen stehen infizierte Schweine sowie Ratten und Mäuse im Vordergrund. Letztere können den Erreger bis zu 180 Tage lang ausscheiden (Heinritzi, 2002). Aber auch Personen sowie Vögel oder Fliegen können als Vektoren B. hyodysenteriae übertragen. Um das Risiko einer Krankheitseinschleppung in einen Betrieb zu minimieren, sind Biosicherheitsmassnah- 
men und eine strategische Bekämpfung von Fliegen und Schadnagern von zentraler Bedeutung (Vyt et al., 2012). Der Anteil von B. hyodysenteriae-infizierten Beständen liegt weltweit zwischen $0 \%$ (Jacobson et al., 2005) und $45 \%$ (Biksi et al., 2007). In der Schweiz sind gemäss Mitteilung des Schweinegesundheitsdienstes (SGD) aktuell etwa 60 Betriebe (knapp 2\% aller SGD-Betriebe) mit B. hyodysenteriae bedingtem Durchfall bekannt. Da nicht alle Schweinebetriebe beim SGD angeschlossen sind und Durchfallproblembetriebe bisher nicht systematisch auf Brachyspira spp. untersucht wurden, muss mit einer Dunkelziffer gerechnet werden. Gemäss SGD müssen Jungsauenproduzenten bei Vorliegen von Klinik und Nachweis von B. hyodysenteriae zwingend sanieren. Da das Platzieren von B. hyodysenteriae-infizierten Mastferkeln den Vermarktungsorganisationen immer grössere Schwierigkeiten bereitet, und sich die Erkrankung auch mit Langzeitantibiotika nicht tilgen lässt (Heinritzi, 2002), steht eine Sanierung der verlustreichen Krankheit im Vordergrund. Aufgrund der tiefen Prävalenz in SGD-Beständen sind die Voraussetzungen günstig, mit Sanierungen der grossen Verbreitungsgefahr durch den Handel mit infizierten Tieren entgegenzuwirken. Dadurch kann der Antibiotikaeinsatz reduziert und auch die damit verbundene Antibiotikaresistenzproblematik entschärft werden, was besonders in Bezug auf B. hyodysenteriae wichtig ist, da nur wenige Antibiotika wirksam sind. In Deutschland fand Schlez (2011) Resistenzraten von B. hyodysenteriae gegenüber Tylosin von $100 \%$, Lincomycin $65 \%$, Tiamulin $4 \%$ und Valnemulin $0 \%$. Deutlich höhere Resistenzraten ergab eine Untersuchung von Rohde (2007) mit Werten für Tylosin von $100 \%$, Lincomycin $98 \%$, Tiamulin $49 \%$ und Valnemulin $30 \%$. Verschiedene Berichte über die $\mathrm{Zu}$ nahme der minimalen Hemmkonzentration von B. hyodysenteriae gegenüber den Pleuromutilinen (Tiamulin bzw. Valnemulin) geben in jüngster Zeit Anlass zur Besorgnis (Lobova et al., 2004; Hidalgo et al., 2011).

Während in einigen Ländern schon einige Dysenteriesanierungen mit Erfolg durchgeführt wurden (Waldmann, 1992; Szancer, 2005), liegen in der Schweiz einzig Erfahrungen in einem Mastbetrieb vor (Speiser et al., 2011). Ziel der vorliegenden Arbeit war es, eine rollende Dysenteriesanierung, bei der sanierte und noch nicht sanierte Schweine gleichzeitig auf dem Betrieb gehalten wurden, durchzuführen und zu dokumentieren, um Grundlagen für weitere Sanierungen zu erhalten. Dabei galt es den Erfolg des Sanierungskonzeptes zu überprüfen, den Effekt der Sanierung anhand von Leistungsverbesserungen zu messen und die Sanierungskosten zu berechnen.

\section{Tiere, Material und Methoden}

\section{Vorgeschichte}

Im Spätsommer 2010 wurde an der Eberaufzuchtstation in Sempach bei einem Eber mit Durchfall B. hyodysen- teriae nachgewiesen. Bei der darauffolgenden epidemiologischen Abklärung konnte im vorliegenden Betrieb ebenfalls B. hyodysenteriae gefunden werden. Der Betrieb hatte in den Ferkelaufzuchtställen gelegentlich mit nicht blutigen Durchfällen zu kämpfen. Wegen des Nachweises von B. hyodysenteriae und der gelegentliche klinischen Symptomatik wurde der Gesundheitsstatus des Kernzuchtbetriebes durch den SGD mit dem Vermerk «B. hyo» versehen. Wollte der Betriebsleiter weiterhin am Jungsauenmarkt teilhaben, musste auf dem Betrieb eine Dysenteriesanierung durchgeführt werden.

\section{Betrieb}

Der Kernzuchtbetrieb mit 170 Zuchtschweinen und 600 Jungsauenaufzucht- und Mastplätzen erfüllte die höchsten Hygiene- und Biosicherheitsanforderungen des SGD. Es wird im 3-Wochen-Rhythmus gearbeitet. Die Schweine sind in 2 Gebäuden untergebracht (Abb. 1). Die Güllekanäle der verschiedenen Stallkammern sind nicht miteinander verbunden und können somit einzeln gespült werden. Unterhalb des Gebäudes 2 befinden sich zwei grosse Güllegruben, die bei Bedarf in den ausserhalb der Gebäude liegenden Güllesilo entleert werden können. Die beiden Stallgebäude sind mit einem hohen Zaun umgeben. Der Personenzugang erfolgt über eine Hygieneschleuse mit Kleider- und Stiefelwechsel. Fliegen und Schadnager werden seit jeher regelmässig bekämpft. Während sich die Fliegenbekämpfung bereits auf hohem Niveau befand, gab es bei der Schadnagerbekämpfung noch Verbesserungspotenzial.

\section{Sanierungskonzept}

Um die landesweit bekannte Sauengenetik erhalten zu können, kam eine Keulung des Bestandes (Totalsanierung) nicht in Frage, sondern nur eine Teilsanierung. Im Unterschied zu herkömmlichen Sanierungsempfehlungen wurde bei der vorliegenden Sanierung sowohl auf eine saugferkelfreie Periode (Waldmann, 1992) als auch auf ein vollständiges jungtierfreies Intervall von Schweinen zwischen 15-70 kg Körpergewicht (KGW) (Szancer, 2005) verzichtet, da einerseits alle Absetzferkel, die während der Sanierung in noch nicht gereinigten Abferkelkammern geboren wurden, direkt nach dem Absetzen verkauft wurden und andererseits die Herdenverjüngung aufrechterhalten werden sollte. Zu Beginn der Sanierung wurden eine Stallskizze mit dem Verlauf der Güllekanäle sowie die wichtigsten Managementdaten aufgenommen. Das Sanierungskonzept mit Zeitplan und den durchzuführenden Arbeitsschritten wurde mit dem Betriebsleiter, dem Bestandestierarzt und externen Fachleuten vor Ort besprochen.

Die Sanierung dauerte von Mitte April bis Anfang Juli 2011 (12 Wochen). Vor Beginn der Sanierung wurde die Schadnager- und Fliegenbekämpfung intensiviert. Anschliessend wurde der Betrieb in einer ersten Phase 


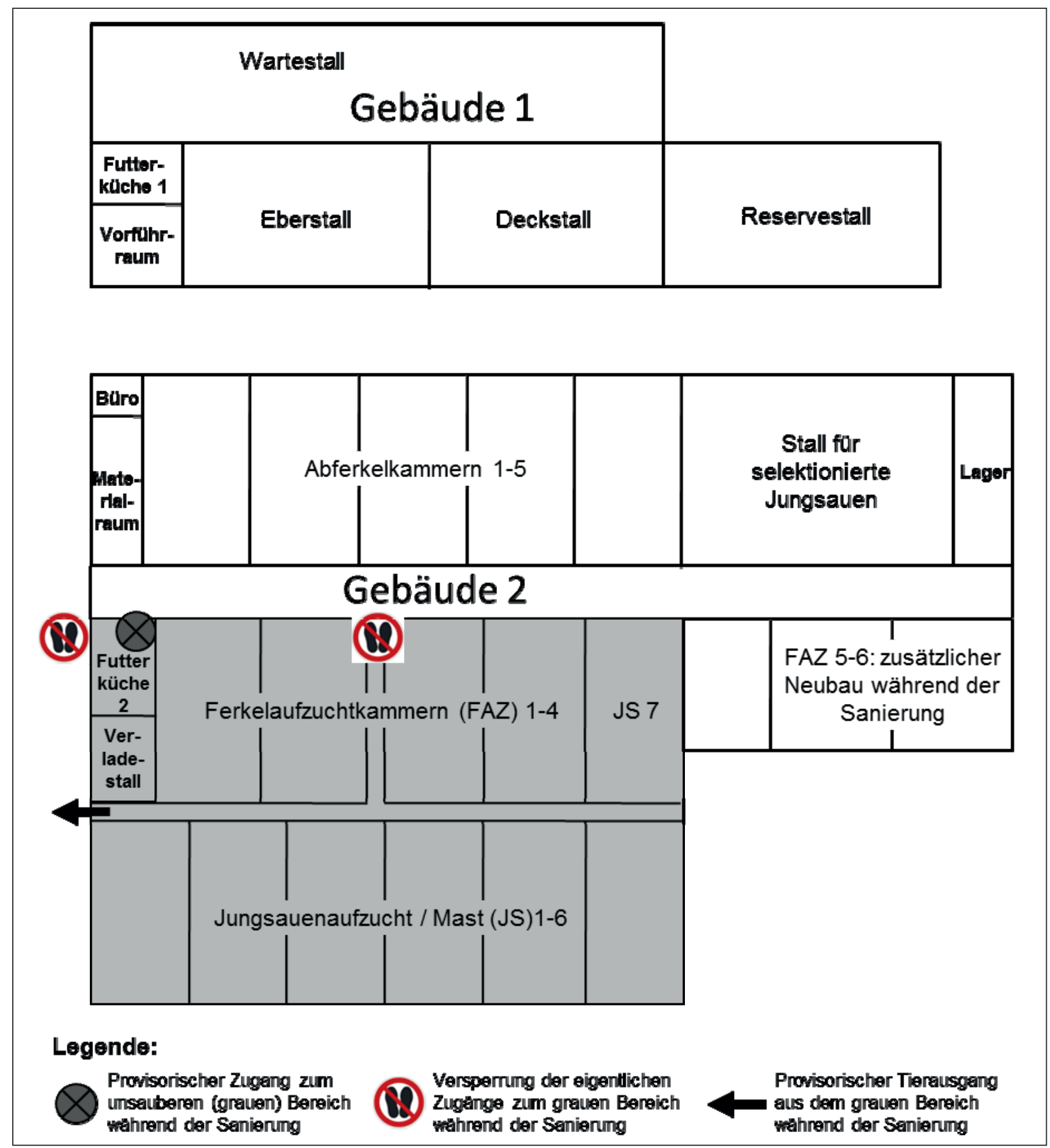

Abbildung 1: Skizze der beiden Stallgebäude bei Sanierungsbeginn: Die Betriebsanlage wurde in einen weissen («sauberen») und einen grauen («unsauberen») Bereich unterteilt.

mittels Absperrungen und Hygieneschleusen in einen «sauberen» und einen "unsauberen» Bereich unterteilt (Abb. 1). Alle Sauen im «sauberen» Bereich wurden mit Ausnahme der Saugferkel während mindestens 4 Wochen mit $8.1 \mathrm{mg}$ Tiamulin pro $\mathrm{kg} \mathrm{KGW}$ und Tag (entspricht $100 \mathrm{mg}$ Denagard ${ }^{\circledR}$ Premix $10 \%$, Novartis Tiergesundheit AG, CH-4058) peroral behandelt.

Zwölf Tage nach Beginn der peroralen Therapie wurden in einer zweiten Phase die Stallungen in 4 Etappen vom
Wartestall bis zu den Jungsauenaufzucht-/Mastkammern gemäss Ablauf in Tabelle 1 gereinigt. Im sauberen Bereich verblieben nur behandelte Sauen und deren neugeborene Ferkel, sowie behandelte Nachzuchttiere. Alle Schweine im unsauberen Bereich wurden entweder als Aufzuchtferkel verkauft oder ausgemästet und dann geschlachtet. Die Abferkelkammern wurden jeweils während der einwöchigen Leerzeit, welche im Produktionsrhythmus entstanden ist, gereinigt und desinfiziert. Die letzte Etappe 
beinhaltete die Sanierung des unsauberen Bereiches, welcher bis zu diesem Zeitpunkt geleert wurde, womit das Waschen der Schweine entfiel. Zwischen den verschiedenen Etappen verhinderten zusätzlich eingerichtete Hygieneschleusen eine Rekontamination der bereits gereinigten Stallabteile durch das Stallpersonal. Ganz am Schluss der Sanierung wurden auch noch die beiden Güllegruben unter dem grossen Gang soweit möglich entleert und die Restgülle mit Alzogur ${ }^{\circledR}\left(3 \mathrm{l} / \mathrm{m}^{3}\right)$ behandelt.

\section{Kotuntersuchung und Überwachung des Sanierungserfolges}

Kotproben wurden entweder am Zentrum für Zoonosen, bakterielle Tierkrankheiten und Antibiotikaresistenz (ZOBA) in Bern mittels direkter real-time PCR auf $B$. hyodysenteriae untersucht (Bürki et al., 2011) oder am Institut für Veterinärbakteriologie der Universität Zürich mittels BJ-Actidion Agar (Dünser et al., 1997) ohne Cyclohexidin kultiviert und anschliessend mittels PCR (La et al., 2003) nach Spezies differenziert. Zur Verifizierung des Sanierungserfolges wurden während 6 Monaten alle 2 Monate je 10 Kottupfer von je 10 älteren Absetzferkeln mit Durchfall untersucht.

\section{Kosten der Sanierung}

Die Sanierungskosten setzen sich vor allem aus den direkten Kosten (Tierarzneimittel, Alzogur ${ }^{\circledR}$, Desinfektionsmittel, Arbeitsaufwand) und dem sanierungsbedingten Ertragsausfall zusammen. Zur Berechnung der Arbeitskosten wurde ein Stundenlohn von CHF 25.- eingesetzt. Wegen der Sanierung mussten rund 500 Ferkel direkt nach dem Absetzen verkauft werden. Zur Berechnung des Ertragsausfalles wurde mit einem Deckungsbeitrag (DB) von CHF 20.- pro Ferkel gerechnet (Angabe der Vermarktungsorganisation). Für die Sanierung musste der Maststall geleert werden. Zur Berechnung der An-

Tabelle 1: Arbeitsschritte bei der Reinigung und Desinfektion der Stallungen.

\begin{tabular}{|c|c|}
\hline Schritt & Arbeitsschritte \\
\hline 1 & Frühmorgens: Schweine ausstallen. \\
\hline 2 & Einweichung und gründliche Reinigung der Stallkammern. \\
\hline 3 & $\begin{array}{l}\text { Entmistung/Entleerung der Güllekanäle } \\
\text { inkl. Zerstörung der Schwimmschicht. }\end{array}$ \\
\hline 4 & $\begin{array}{l}\text { Restgüllebehandlung mit Cyanamid (Alzogur }{ }^{\circledR}, 31 / \mathrm{m} 3 \\
\text { Restgülle, AlzChem AG, D-83308 Trostberg,). }\end{array}$ \\
\hline 5 & Abtrocknung der Kammer mit Grossraumlüfter. \\
\hline 6 & $\begin{array}{l}\text { Desinfektion mit Venno Vet } 1 \text { Super }{ }^{\circledR}(1.5 \%, 1 \mathrm{~h} \text { Einwir- } \\
\text { kungszeit, Vital AG, CH-5036 Oberentfelden }) \text {. }\end{array}$ \\
\hline 7 & $\begin{array}{l}\text { Waschen der Sauen mit Sauenwaschmittel } \\
\text { (BestFarm }{ }^{\circledR}, 2 \% \text {, SUISAG, CH-6204 Sempach). }\end{array}$ \\
\hline 8 & $\begin{array}{l}\text { Einstallung der sauberen Schweine in die gereinigten } \\
\text { und desinfizierten Stallungen. }\end{array}$ \\
\hline
\end{tabular}

zahl Mastschweine, die während der Sanierungsphase hätten ausgemästet werden können, wurden sämtliche Leertage (Leerzeit $\times$ Mastplätze) zusammengezählt und dann durch 110 Tage (Annahme einer durchschnittlicher Mastdauer eines Mastschweines) dividiert. Dieser Ausfall wurde mit einem DB von CHF 63.- pro Mastschwein (AGRIDEA, 2011) berechnet. Zusätzliche Kosten für den Ersatz von Gerätschaften und Stallkleidern sowie für bauliche Massnahmen wurden pauschal geschätzt.

\section{Nutzen der Sanierung}

Um eine Aussage über den Einfluss der Sanierung auf die Leistung der Tiere zu erhalten, wurden die durchschnittlichen Lebendtageszunahmen (LTZ) der Jungsauen von Geburt bis zur Selektion zwischen Oktober und Dezember 2010 (vor der Sanierung) mit den LTZ von Oktober 2011 bis Januar 2012 (nach der Sanierung) verglichen. Diese Daten wurden mit der Software «R» (R Development Core Team, 2011) und dem package nlme (Pinheiro et al., 2011) ausgewertet. Mit Hilfe eines gemischten linearen Modelles wurde der Effekt der Sanierung und der Rasse berechnet. Die Modellauswahl, in welchem die Effekte Linie, Vater, Mutter und der Wurf sowie eine Interaktion zwischen der Sanierung und der Rasse berücksichtigt wurde, erfolgte nach Akaike's Informationskriterien (AIC).

Zusätzlich wurden aufgrund der Leistungsdifferenz einerseits Mastdauer und der dadurch beeinflusste Umsatz an Mastschweinen sowie der zusätzliche Futterverbrauch (CHF 0.62 pro kg Futter) pro Jahr errechnet. Es wurde von einem durchschnittlichen Futterverzehr von $1.4 \mathrm{~kg}$ Futter pro Tier und Tag ausgegangen.

Ohne Sanierung hätte der Betrieb die Jungsauenproduktion aufgeben und auf die Produktion von Mastferkeln umstellen müssen, was mit einer Reduktion des Deckungsbeitrages von CHF 125.- pro Jungsau verbunden gewesen wäre (Angabe der Vermarktungsorganisation). Zusätzlich wurden Behandlungskosten für eine 3-wöchige Behandlung von CHF 20.- pro Jungsau für rund 100 verkaufsfertige Jungsauen, die während der halbjährigen Übergangszeit von der Bestätigung der Dysenterie bis zur Sanierung behandelt werden mussten, miteingerechnet.

\section{Ergebnisse}

\section{Labor}

Die Kotuntersuchungen vor und nach der Sanierung sind in Tabelle 2 dargestellt. Vor der Sanierung wurden in 25\% der Proben (21/84) B. hyodysenteriae nachgewiesen, wobei in drei von vier Untersuchungszeitpunkten positive Kotproben gefunden wurden. Bei der negativen Untersuchung vom Januar 2011 wurden keine Durchfalltiere auf dem Betrieb vorgefunden. Die Untersuchungen ab Sep- 
Tabelle 2: Resultate der auf B. hyodysenteriae untersuchten Kotproben. Sanierungszeitpunkt (--). ${ }^{1}$ Alle Tiere ohne Durchfall. ${ }^{2}$ Pro Probe wurden 2 Schweine gepoolt.

\begin{tabular}{|l|c|c|}
\hline $\begin{array}{l}\text { Kotproben- } \\
\text { entnahmedatum }\end{array}$ & Laborort & Positive/Total Proben \\
\hline 06.09 .2010 & BE & $8 / 36$ \\
\hline 20.09 .2010 & ZH & $11 / 13$ \\
\hline $20.01 .2011^{1}$ & ZH & $0 / 30$ \\
\hline 13.04 .2011 & ZH & $2 / 5$ \\
\hline 05.09 .2011 & ZH & $0 / 10$ \\
\hline 07.11 .2011 & ZH & $0 / 10$ \\
\hline 04.01 .2012 & ZH & $0 / 10$ \\
\hline $23.07 .2012^{2}$ & ZH & $0 / 3$ \\
\hline $11.02 .2013^{2}$ & ZH & $0 / 3$ \\
\hline
\end{tabular}

tember 2011 dienten zur Überprüfung des Sanierungserfolges und waren seither immer negativ.

\section{Kosten der Sanierung}

Die Sanierungskosten sind in Tabelle 3 aufgeführt. Total beliefen sich die gesamten Sanierungskosten auf etwa CHF 104'500.-. Rund die Hälfte der Kosten wurde durch die Sanierung direkt verursacht (Tierarzt/Medikamente, Desinfektionsmittel, Schadnagerbekämpfung, Wasserverbrauch, Arbeitsaufwand, Diagnostikkosten). Der sanierungsbedingte Ertragsausfall durch Nicht-Auslastung der Stallungen machte ca. $40 \%$ der Sanierungskosten aus. Schliesslich wurden die restlichen $10 \%$ der Kosten für den Ersatz von Gerätschaften und Stallkleidern und für bauliche Massnahmen nach der Sanierung verwendet. Zum Letzteren gehörten das Zumauern von allfälligen Nistplätzen und Schlupflöcher für Schadnager mit Mörtel sowie das Malen von Wänden für eine bessere Reinigung.

\section{Nutzen der Sanierung}

Aus der Periode vor der Sanierung wurden 209 Feldmessungsdaten von 104 Edelschweinen (ES) und 105 Kreuzungsauen (F1) mit 217 Feldmessungsdaten, welche von 102 ES und von 115 F1 nach der Sanierung stammten, verglichen. In der ersten Zeile in Tabelle 4 sind die mithilfe des statistischen Models erhaltenen Werte der LTZ aufgeführt. Die Auswertung zeigte eine signifikante $(\mathrm{P}<0.0001)$ um 23.8 g ( $\pm 10.1 \mathrm{~g})$ höhere LTZ nach der Sanierung. Dieser Effekt der Sanierung war rasseunabhängig.

Durch die höhere LTZ von $23.8 \mathrm{~g}$ nach der Sanierung verkürzt sich die Mastdauer rein rechnerisch bei gleichem Endgewicht von $105 \mathrm{~kg}$ um 6.5 Tage. Bei 600 Mastplätzen können dadurch jährlich 119 Tiere mehr ausgemästet werden, was einen CHF 7'497.- höheren Deckungsbeitrag ergibt (Tab. 4). Bei einer um 6.5 Tage verkürzten Mastdauer können theoretisch $9.2 \mathrm{~kg}$ Futter pro Mastschwein eingespart werden, wodurch sich die Futterkosten pro Tier um CHF 5.68 reduzieren. Bei 1'938 ausgemästeten Schweinen pro Jahr ergibt dies eine Reduktion der Futterkosten von CHF 11'000.-. Insgesamt erbrachte die Sanierung somit einen geschätzten finan-

Tabelle 3: Zusammenstellung der Kosten der rollenden Dysenteriesanierung.

\begin{tabular}{|c|c|c|}
\hline Kostenpunkt & Menge & Kosten in CHF \\
\hline Strategische Schadnagerbekämpfung & 94 Köder & 395.- \\
\hline Medikation mit Denagard ${ }^{\circledR}$ Premix $10 \%$ & $340 \mathrm{~kg}$ & $25^{\prime} 024 .-$ \\
\hline Tierarztkosten & 15 Std. & 3'300.- \\
\hline Desinfektion mit Venno Vet 1 Super ${ }^{\circledR}$ & 401 & 860.- \\
\hline Gülledesinfektion mit Alzogur ${ }^{\circledR}$ & $1^{\prime} 1201$ & 8'614.- \\
\hline Sauenwaschmittel BestFarm ${ }^{\circledR}$ & 101 & $58 .-$ \\
\hline Arbeit für Reinigung und Desinfektion & 510 Std. & $12^{\prime} 750 .-$ \\
\hline Wasser für Reinigung und Desinfektion & $500 \mathrm{~m}^{3}$ & 795.- \\
\hline Kotproben während Überwachung & $3 \times 10$ Stk. & 800.- \\
\hline Ersatz von Gerätschaften und Stallkleidern & Pauschal & 1'000.- \\
\hline Bauliche Sanierungsmassnahmen (Material) & Pauschal & 5'000.- \\
\hline Bauliche Sanierungsmassnahmen (Arbeit) & 200 Std. & $5^{\prime} 000 .-$ \\
\hline Verlust an nicht aufgezogenen Ferkeln in den Ferkelaufzuchtställen aufgrund Leerzeit & 500 Tiere & 10'000.- \\
\hline Verlust an nicht ausgemästeten Schweinen in den Jungsauenaufzuchtställen aufgrund Leerzeit & 357 Tiere & $222^{\prime} 491 .-$ \\
\hline $\begin{array}{l}\text { Verlust an nicht ausgemästeten Schweinen in den Ställen für selektionierte Jungsauen } \\
\text { aufgrund reduzierter Stallauslastung }\end{array}$ & 134 Tiere & $8^{\prime} 442 .-$ \\
\hline Total & & 104'529.- \\
\hline
\end{tabular}


ziellen Nutzen durch Leistungsverbesserung von jährlich CHF 18'497.-.

Der Nachweis von B. hyodysenteriae bedeutete für den Betrieb eine finanzielle Einbusse von CHF 62'500.- pro Jahr, da die Nachfrage von jährlich 700 auf 200 Jungsauen zurückging. Ohne Sanierung wäre mit der Aufgabe der Jungsauenverkäufe ein weiterer jährlicher Verlust von CHF 25'000.- dazugekommen. Zusammen mit der Leistungsverbesserung konnten dank der Sanierung die Einbussen im Betrieb um rund CHF 43'500.- pro Jahr vermindert werden, so dass die Sanierungskosten von CHF 104'500.- und die vorgängigen Behandlungskosten für die Jungsauen von CHF 2'000.- in rund 2.5 Jahren amortisiert werden können.

\section{Diskussion}

Eine Dysenteriesanierung, bei der die Erreger gleichzeitig im Tier und in der Umgebung des Tieres bekämpft werden, ist als einzige effektive Bekämpfungsmassnahme anzusehen. Die Erfolgsquote von Dysenteriesanierungen wird auf $54 \%$ und bei sehr gut geführten Betrieben bis $90 \%$ geschätzt (Wood und Lysons, 1988). Trotz intensiver Diagnostik kann keine 100\%-ige Erregerfreiheit garantiert werden, da asymptomatische Schweine nur gelegentlich eine detektierbare Erregermenge im Kot (>103 Zellen $/ \mathrm{ml}$ ) ausscheiden (Hampson, 2012). Durch eine Sanierung findet aber zumindest eine massive Erregerverdünnung statt, so dass die Tiere symptomfrei und somit unverdächtig für die Dysenterie sind. Akut erkrankte Tiere scheiden hingegen grosse Mengen von
B. hyodysenteriae $\left(10^{8}-10^{9} / \mathrm{g} \mathrm{Kot}\right)$ aus (Hampson, 2012). Daher können durch die Beprobung von Durchfalltieren sowohl die Probenzahl wie auch die Diagnostikkosten reduziert werden. Wie die Resultate von Kotuntersuchungen vor der Sanierung zeigen, konnte B. hyodysenteriae immer gefunden werden, wenn Durchfalltiere beprobt wurden. Während die vom SGD vorgesehene halbjährliche Routineüberwachung mit 3 gepoolten Kotproben von 6 Durchfalltieren eher an der unteren Grenze liegt, hätte zumindest in der intensiven Überwachungsphase im ersten halben Jahr nach der Sanierung eine Reinfektion mit grosser Wahrscheinlichkeit entdeckt werden müssen.

Die hier dargestellte Sanierung kann als kostengünstigste Variante angesehen werden. Im Zuchtbereich fielen etwa $65 \%$ der Kosten an und im Jungsauenaufzucht-/ Mastbereich die restlichen $35 \%$. Die Behandlungskosten entstanden ausschliesslich im Zuchtbereich und machten mit knapp 40 \% den grössten Anteil aus. Im Jungsauenaufzucht-/Mastbereich fielen v.a. die Leertage mit einem Anteil von 60 \% ins Gewicht. In der Literatur kann einzig eine einfache Kostenaufstellung für die Sanierung eines 270 grossen Zuchtschweinebetriebes gefunden werden (Wood und Lysons, 1988). Im Vergleich zu unserer Sanierung wurde dort der Bestand während der Sanierung nicht reduziert, weshalb mehr Tiere behandelt werden mussten und deshalb die Behandlungskosten mit knapp $90 \%$ einen noch höheren Kostenfaktor bildeten.

Bei der Berechnung der Futterkosteneinsparung wurde der Futterverzehr nur geschätzt. Weil kranke Schweine normalerweise weniger fressen als gesunde, dürfte die Futtereinsparung durch die Sanierung überschätzt sein, jedoch nur leichtgradig, da die Klinik auf dem Betrieb nicht sehr

Tabelle 4: Berechnung des Einflusses einer um $23.8 \mathrm{~g}$ höheren Lebendtageszunahme (LTZ) nach der Sanierung auf den Mehrerlös durch höheren Tierumsatz.

\begin{tabular}{|c|c|c|}
\hline Ganze Lebensdauer & Vor der Sanierung & Nach der Sanierung \\
\hline LTZ in g bezogen auf Standardgewicht von $97.5 \mathrm{~kg}$ & 593.5 & 617.3 \\
\hline LTZ in g korrigiert (auf Endgewicht von $105 \mathrm{~kg}$ ) & 606.2 & 630.0 \\
\hline Tage bis $105 \mathrm{~kg}$ & 173.2 & 166.7 \\
\hline \multicolumn{3}{|l|}{ Ferkelaufzuchtphase } \\
\hline Tage Saugferkelphase (Annahme) & 27.0 & 27.0 \\
\hline Tage Ferkelaufzucht (Annahme) & 43.2 & 43.2 \\
\hline Tageszunahme Ferkelaufzucht in $g$ & 393.5 & 418.0 \\
\hline Gewicht bei 70.5 Tagen in $\mathrm{kg}$ & 25.0 & 26.1 \\
\hline \multicolumn{3}{|l|}{ Mastphase } \\
\hline$\varnothing$ Mastdauer (Einstallen bis $105 \mathrm{~kg}$ ) in Tagen & 103.0 & 96.5 \\
\hline Tageszunahme Mast in $\mathrm{g}$ & 776.7 & 818.3 \\
\hline Umtriebsdauer in Tagen & 113.0 & 106.5 \\
\hline Anzahl Umtriebe pro Jahr & 3.23 & 3.43 \\
\hline Ausgemästete Schweine pro Jahr bei 600 Plätzen & $1^{\prime} 938$ & $2^{\prime} 057$ \\
\hline Deckungsbeitrag pro Jahr in CHF (CHF 63.-/Tier) & $122^{\prime} 094 .-$ & $129 ' 591 .-$ \\
\hline Mehrerlös durch höheren Tierumsatz in CHF/Jahr & & 7'497.- \\
\hline
\end{tabular}


ausgeprägt war. Aus dem gleichen Grund fallen die Medikamentenkosten vor der Sanierung nur unwesentlich ins Gewicht. Die Behandlung der Jungsauen unmittelbar vor dem Verkauf geschah nicht aufgrund von klinischen Symptomen, sondern prophylaktisch zur Minimierung des Verschleppungsrisikos und zur Aufrechterhaltung der Jungsauenverkäufe für die bestehende Kundschaft.

Betrachtet man den vorliegenden Betrieb nur als Zuchtmastbetrieb ohne Jungsauenproduktion, so steht den Sanierungskosten von knapp CHF 104'500.- ein Sanierungsgewinn durch Leistungsverbesserung von jährlich etwa CHF 18'500.- gegenüber. Unter der Voraussetzung, dass diese Leistungssteigerung langfristig aufrechterhalten werden kann, ist die Sanierung nach rund 5.5 Jahren abgeschrieben. Wood und Lysons (1988) berichten, dass sich eine Dysenteriesanierung bereits nach einem Jahr auszahlt. Der grosse Unterschied in der Amortisationsdauer kann damit erklärt werden, dass unser Sanierungsbetrieb bereits auf einem hohen Leistungsniveau arbeitete und Symptome der Dysenterie im Betrieb in erster Linie subklinisch waren.

\section{Assainissement partiel modifié de la dysente-} rie dans une exploitation d'élevage de porc

On a réalisé en 2011 dans une exploitation suisse d'élevage de porcs positive à Brachyspira (B.) hyodysenteriae et comptant 170 truies d'élevage et 600 jeunes truies de remonte un assainissement par roulement sur une durée de 12 semaines. Outre un traitement oral de 4 semaines ( $8.1 \mathrm{mg}$ de Tiamulin par kg de poids corporel) appliqué aux animaux restant sur l'exploitation, on a procédé à un nettoyage approfondi des locaux et on a désinfecté le lisier restant avec de l'Alzogur $^{\circledR}\left(3 \mathrm{l} / \mathrm{m}^{3}\right)$ et les surfaces de la porcherie avec du Venno Vet 1 Super $^{\circledR}(1.5 \%)$. Parallèlement on a intensifié la lutte contre les rongeurs et les mouches. Suite à cet assainissement, on a surveillé l'exploitation durant 6 mois au moyen d'écouvillons de selles prélevés chez les animaux atteints de diarrhées. On n'a plus alors constaté la présence de $B$. hyodysenteriae dans aucun échantillon. Les coûts de cet assainissement se sont élevés à environ CCHF 104'500.-. Ces mesures ont conduit à une prise de poids journalière significativement plus élevée $(+23.8$ g, \pm 10.1 g, P < 0.0001). Cette amélioration de la productivité amenait un gain annuel supplémentaire de CHF 18'500.-.

\section{Schlussfolgerungen}

Unsere Ergebnisse haben gezeigt, dass eine rollende Dysenteriesanierung unter Praxisbedingungen erfolgreich durchgeführt werden kann und finanziell vertretbare Verluste verursacht. Sie stellt jedoch an die Planung und Durchführung höchste Anforderungen. Es ist von zentraler Bedeutung, dass genügend Platz und motiviertes Personal vorhanden ist und dass sämtliche Biosicherheitsmassnahmen verstärkt werden, um das Risiko für eine Reinfektion zu minimieren. Der Gesundheitsstatuts kann durch eine Dysenteriesanierung verbessert und das Leistungsniveau gesteigert werden.

\section{Dank}

Ein herzlicher Dank geht an die Novartis Tiergesundheit AG, CH-4058 Basel, und die AlzChem AG, D-83308 Trostberg, welche uns tatkräftig unterstützt haben und beratend zur Seite standen, sowie an das Betriebsleiterehepaar und an die Vermarktungsorganisation Prosus.

\section{Eradicazione della dissenteria suina con spopolamento parziale in un'azienda di allevamento intensivo di suini}

Nel 2011, in un'azienda di allevamento intensivo di suini con 170 scrofe e 600 giovani suini di allevamento/da ingrasso, dopo il rilevamento nella mandria di Brachyspira (B.) hyodysenteriae è stato condotto un trattamento di eradicazione della dissenteria con spopolamento parziale su un periodo di 12 settimane. Oltre alla somministrazione di un farmaco orale durante quattro settimane $(8,1 \mathrm{mg}$ di tiamulina per $\mathrm{kg}$ di peso corporeo) ai suini rimasti nell'azienda, venivano accuratamente pulite tutte le stalle e il liquame residuo veniva disinfettato con Alzogur ${ }^{\circledR}\left(31 / \mathrm{m}^{3}\right)$ mentre le superfici delle stalle con Venno Vet 1 Super ${ }^{\circledR}(1.5 \%)$. Allo stesso tempo, era stato intensificato il controllo dei roditori e delle mosche. Dopo l'eradicazione l'azienda è stata controllata regolarmente durante 6 mesi con analisi di tamponi di feci degli animali con diarrea. Tutti i campioni di feci erano risultati negativi a B. hyodysenteriae. I costi per l'eradicazione ammontavano a circa CHF 104'500.-. L'eradicazione ha comportato un guadagno significativo di peso giornaliero vivo (+23.8 g $\pm 10.1 \mathrm{~g}, \mathrm{p}<0,0001)$. Questo miglioramento della prestazione ha indotto un beneficio economico annuale di CHF 18'500.-. 


\section{Literatur}

AGRIDEA: Deckungsbeiträge Ausgabe 2011: 34 (CCM, $850 \mathrm{~g}$ Tageszunahme).

Biksi I., Lorincz M., Molnar B., Kecskes T., Takacs N., Mirt D., Cizek A., Pejsak Z., Martineau G. P., Sevin J.-L., Szenci O.: Prevalence of selected enteropathogenic bacteria in Hungarian finishing pigs. Acta. Vet. Hung. 2007, 55: 219-227.

Boye M., Baloda S. B., Leser T. D., Möller K.: Survival of Brachyspira hyodysenteriae und B. pilosicoli in terrestrial microcosms. Vet. Microbiol. 2001, 81: 33-40.

Bürki S., Vilei E. M., Frey J., Wittenbrink, M. M.: Allelic variations of the nox gene of Brachyspira pilosicoli impair its detection by qPCR. Vet. Microbiol. 2011, 149: 291-292.

Dünser M., Schweighardt H., Pangerl R., Awad-Masalmeh M., Schuh M.: Schweindysenterie und Spirochaetendiarrhoe - vergleichende Untersuchungen serpulinenbedingter Enteritiden. Wien. Tierarztl. Monatsschr., 1997, 84: 151-161.

Hampson D. J.: Brachyspiral Colitis. In Diseases of swine. Hrsg. J. J. Zimmermann, L. A. Karriker, A. Ramirez, K. J. Schwartz, G. W. Stevenson, Wiley-Blackwell-Verlag, 2012, 680-696.

Heinritzi K.: Schweinedysenterie - Bedeutung und Behandlungsmöglichkeiten. Nutztierpraxis Aktuell 2002, 3: 37-39.

Hidalgo A., Carvajal A., Vester B., Pringle M., Naharro G., Rubio P.: Trends towards lower antimicrobial susceptibility and characterization of acquired resistance among clinical isolates of Brachyspira hyodysenteriae in Spain. Antimicrob. Agents Chemother. 2011, 55: 3330-7.

Jacobson M., Löfstedt M. G., Holmgren N., Lundeheim N., Fellström C.: The prevalences of Brachyspira spp. and Lawsonia intracellularis in Swedish piglet producing herds and wild boar population. J. Vet. Med. B 2005, 52: 386-391.

La T., Phillips N. D., Hampson D. J.: Development of a duplex PCR assay for detection of Brachyspira hyodysenteriae und Brachyspira pilosicoli in pig feces. J. Clin. Microbiol. 2003, 41: 3372-5.

Lobova D., Smola J., Cizek A.: Decreased susceptibility to tiamulin and valnemulin among Czech isolates of Brachyspira hyodysenteriae. J. Med. Microbiol. 2004, 53: 287-291.

Pinheiro J., Bates D., DebRoy S., Sarkar D. and the R Development Core Team: nlme: Linear and Nonlinear Mixed Effects Models. 2011, R package version 3.1-102.
$R$ Development Core Team: R: A language and environment for statistical computing. R Foundation for Statistical Computing, Vienna (A), 2011. URL: http://www.R-project.org/.

Rohde J.: Informationen zur Resistenzprüfung von Brachyspiren. http://www.tihohannover.de/fileadmin/user_upload/tiho_ hannover/kliniken_institute/18_mikrobiologie/Info-Dokumente/diagnostik_info_brachyspiren_resi.pdf, 2007.

Schlez K.: Bestimmung der Antibiotikaempfindlichkeit bei Brachyspira spp.-Stämmen von deutschen Mastschweinen aus den Jahren 2003-2005. Dissertation, Justus-Liebig-Universität Gießen, 2011.

Szancer J.: Eradication programmes for swine dysentery. Int. Pig Topics 2005, 20: 7-9.

Speiser S. A., Zeeh F., Goy N., Albini S., Zimmermann W., Luginbuhl A.: Die Sanierung der Schweinedysenterie in einem Schweizer Aufzucht-Mastbetrieb. Schweiz. Arch. Tierheilk. 2011, 153: 14-18.

Vyt P., Vandepitte L., Dereu A., Roozen M.: Elimination of swine dysentery on a sigle-site, farrow-to-finish farm using tylvalosin (Aivlosin ${ }^{\circledR}$ ). Proceeding IPVS-Kongress, Korea, 2012: 629.

Waldmann K.-H.: Voraussetzungen und Massnahmen zur Sanierung von Ferkelerzeugerbetrieben mit latenter Schweindysenterie. Tierärztl. Prax. 1992, 20: 159-163.

Wood E. N., Lysons R. J.: Financial benefit from the eradication of swine dysentery. Vet. Rec. 1988, 122: 277-279.

\section{Korrespondenz}

Xaver Sidler

Departement für Nutztiere

Abteilung für Schweinemedizin

Vetsuisse-Fakultät Universität Zürich

Winterthurerstrasse 260

8057 Zürich

Schweiz

xsidler@vetclinics.uzh.ch

Manuskripteingang: 1. Oktober 2013

Angenommen: 3. Februar 2014 


\section{Lebenslauf}

\section{Personalien}

$\begin{array}{ll}\text { Name } & \text { Figi } \\ \text { Vorname } & \text { Rolf } \\ \text { Strasse } & \text { Rathausstrasse 31 } \\ \text { Wohnort } & 6280 \text { Hochdorf LU } \\ \text { Telefon } & 0795546560 \\ \text { Geburtsdatum } & 16.08 .1978 \\ \text { Heimatort } & \text { Glarus Süd } \\ \text { Zivilstand } & \text { Ledig } \\ \text { Aufgewachsen } & \text { Auf Bauernhof im Kanton Glarus }\end{array}$

\section{Ausbildungen}

1985-1991 Primarschule Hätzingen (GL)

1991-1997 Kantonsschule Glarus Typ B

1997-1998 Zwischenjahr mit RS und Sommer 1998 auf der elterlichen Pachtalp ob Schwanden (GL)

1998-2003 Agronomiestudium, Fachrichtung Nutztierwissenschaften an der ETH in Zürich mit folgenden Praktika:

- Im 2000: 6 Monate auf landwirtschaftlichem Betrieb in Bätterkinden (BE).

- Im 2001: 9 Wochen bei Eric Meili, landwirtschaftlicher Betriebsberater am Forschungsinstitut für biologischen Landbau (FiBL).

- Im 2002/2003: 4 Wochen sowie 3-monatige Anstellung als Hilfsassistent am Institut für Nutztierwissenschaften der ETH Zürich.

2007-2013 Veterinärmedizinstudium an der Vetsuisse-Fakultät in Zürich mit folgenden Praktika:

- Im 2011: 4 Wochen in der Nutztierpraxis Casura \& Partner in Oberentfelden (AG).

- Im 2012: 4 Wochen an der Aussenstelle für Epidemiologie der Stiftung Tierärztliche Hochschule Hannover (TiHo) in Bakum (DE).

\section{Berufliche Tätigkeiten}

$01.12 .2003-31.05 .2004$

$01.06 .2004-29.02 .2008$

$25.11 .2007-30.09 .2010$

01.07.2008-31.08.2011

$01.03 .2008-31.01 .2013$

$01.01 .2013-28.02 .2014$
Befristete Anstellung im Bereich Weiterbildung und Beratung bei der Fachstelle Betriebswirtschaft an der Liebegg in Gränichen (AG).

Technischer Berater im Bereich Schweine bei der Provimi Kliba AG in Kaiseraugst (AG).

Aushilfe als Zeitungsverträger bei der Südostschweiz Pressevertrieb AG, Glarus (GL).

Teilzeit-Berater im Technischen Dienst der Firma Willi Grüninger AG, Flums (SG).

Tierpfleger (Wochenend-Dienst und Ferienaushilfe) an der VetsuisseFakultät Zürich am Institut für Parasitologie und an der Abteilung für Schweinemedizin.

Assistent und Doktorand an der Abteilung für Schweinemedizin der Vetsuisse-Fakultät Zürich.

Nutztierpraxis Rudolph, Sulz LU (bis 28.02.14 zu 50 \%, ab 1.3.14 zu $100 \%$ ). 